\title{
Bifurcation Type Change of AC Electrostatically Actuated MEMS Resonators due to DC Bias
}

\author{
Dumitru I. Caruntu and Kyle N. Taylor \\ Mechanical Engineering Department, University of Texas-Pan American, Edinburg, TX 78539, USA \\ Correspondence should be addressed to Dumitru I. Caruntu; caruntud2@asme.org
}

Received 17 September 2013; Accepted 12 March 2014; Published 28 April 2014

Academic Editor: Mohammad Elahinia

Copyright ( 2014 D. I. Caruntu and K. N. Taylor. This is an open access article distributed under the Creative Commons Attribution License, which permits unrestricted use, distribution, and reproduction in any medium, provided the original work is properly cited.

\begin{abstract}
This paper investigates the nonlinear response of microelectromechanical system (MEMS) cantilever resonator electrostatically actuated by applying a soft alternating current (AC) voltage and an even softer direct current (DC) voltage between the resonators and a parallel fixed ground plate. The AC frequency is near natural frequency. This drives the resonator into nonlinear parametric resonance. The method of multiple scales (MMS) is used to solve the dimensionless differential equation of motion of the resonator and find the steady-state solutions. The reduced order model (ROM) method is used to validate the results obtained using MMS. The effect of the soft DC voltage (bias) component on the frequency response is reported. It is shown that the DC bias changes the subcritical Hopf bifurcation into a cyclic fold bifurcation and shifts the bifurcation point (where the system loses stability) to lower frequencies and larger amplitudes.
\end{abstract}

\section{Introduction}

Electrostatically actuated microelectromechanical system cantilever resonators (EA-MEMS-CR) have been explored due to their applications as sensors of small mass objects, such as proteins, viruses, or trace amounts of chemical compounds $[1,2]$. Low power, integration into a microchip design, and low cost are a couple of advantages of EA-MEMS-CRs. Resonator sensors are coated for specimen recognition. The sensing principle is based on specimen particles bonding with the coating and increasing the mass of the EA-MEMSCR. This change in mass causes a change in EA-MEMSCR's natural frequency. If the frequency response to input voltage excitation is known accurately, then the increase in mass can be found from the change in natural frequency. One key feature of EA-MEMS-CRs is the nonlinear dynamics they experience $[3,4]$. It is crucial to accurately predict the nonlinear dynamics of EA-MEMS-CRs as a precursor to sensing applications. Sources of nonlinearities include micro- and nanoscale surface forces [5], fringe effect [6$8]$, and damping $[1,9-12]$. In order to solve the nonlinear differential equations of motion, methods such as reduced order model (ROM) [1, 5-8], Green's function [9], multiple scale or perturbation $[8,12]$, and modal expansion [13], have been used. The steady-state solutions of these systems can be stable and/or unstable. Bifurcation points are points where stability changes. Finding stability change frequencies is necessary to accurately model and take advantage of this phenomenon for sensing purposes.

In order to control the behavior of EA-MEMS-CRs, one uses the properties of the applied voltage (amplitude, frequency) since the physical cantilever characteristics cannot be altered after manufacture. Nonlinear effects of alternating current (AC) and direct current (DC) voltages on such system have been reported in the literature $[1,3,4,8,11,12,14]$. Both $A C$ voltage and DC voltage have been used for EA-MEMS-CR excitation. The resulting bifurcation points have been used for sensing applications [1]. Nonlinear primary, superharmonic, and subharmonic resonances due to AC and DC actuation have been reported in [3]. If only DC voltage is applied, a static microbeam deflection is achieved. If both AC and DC are applied, then the structure vibrates with possibility of going into resonance [4]. Changes to the DC voltage have been shown to drastically alter the behavior of the systems 
studied, with small changes shifting a softening response to a hardening response [15]. The softening response consists of a frequency decrease as the amplitude of oscillation increases, whereas the hardening response consists of a frequency increase of the structure as the amplitude of oscillation increases [16]. Nonlinear behavior of electrically actuated MEMS resonator based on the modified couple stress theory, in which the microbeam has been excited by an AC voltage superimposed on a DC voltage, has been reported in the literature [17]. A high-dimensional reduced order model of the continuous system has been obtained by applying Galerkin method. The pseudo-arclength continuation technique has been employed to examine the nonlinear static and dynamic behavior. Frequency and voltage responses [1821] have been reported for EA-MEMS-CRs for soft AC near half natural frequency. This resulted into primary resonance of the system. Nonlinear responses and dynamics of EAMEMS resonant sensors under two-frequency parametric and external excitations have been reported [22]. The investigation has been conducted using the method of multiple scales (MMS). Parametric resonance amplification has been proposed [23] as mass sensing concept and experimentally investigated using a noninterdigitated comb-finger driven microoscillator. Due to the sharp transition in amplitude caused by parametric resonance, the sensitivity has been 1-2 orders of magnitude higher than the same oscillator working at simple harmonic resonance. Dynamic response due to parametric excitation produced by fluctuating voltages of a class of electrostatically driven microelectromechanical comb drives has been investigated [24]. It has been shown that these systems can exhibit not only hardening and softening nonlinearities, but also mixed nonlinearities. Fabrication and testing of MCR subjected to AC and DC voltages have been carried out [14]. Recently, there has been an emphasis on researching the response of nanoscale cantilevers [5, $8,25]$. However, the forces involved in these applications are identical in most cases to a microcantilever, with the exception of small scale forces such as Casimir effect.

This paper investigates the effects of soft DC bias on the nonlinear dynamics of EA-MEMS-CRs under soft AC voltage of frequency near natural frequency [26, 27] of the cantilever. Forces taken into account are due to electrostatic actuation, fringe effect, damping, and beam stiffness. MMS and ROM [18-21] are used to investigate the resulting nonlinear parametric resonance. It is showed that the subcritical Hopf bifurcation, due to only soft AC, becomes a cyclic fold bifurcation due to the DC bias. Soft AC is the voltage that produces soft electrostatic forces in the system; that is, it produces small to very small amplitudes (with respect to the gap between EA-MEMS-CR and the ground plate) when the frequency is away from resonance zones. Effects of system parameters on the frequency response are then investigated. To the best of our knowledge, this is the first time when such change from subcritical Hopf bifurcation to cyclic fold bifurcation due to soft DC bias of soft AC near natural frequency electrostatically actuated MEMS is reported. As mentioned afterwards this work can be used to detect the level of unwanted DC bias in a system or increase the escape band of EA-MEMS-CR.

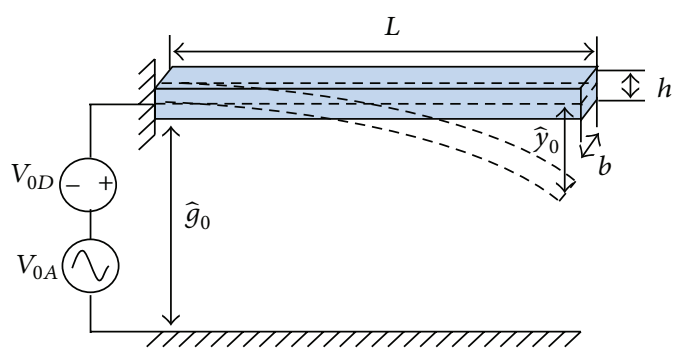

Figure 1: System description.

\section{Equation of Motion}

A system consisting of a uniform MEMS cantilever over a ground plate, Figure 1, is investigated. A soft voltage having both components of DC and AC is applied between the cantilever and the ground plate. The partial differential equation of motion of the cantilever is as follows [20, 21]:

$$
\begin{aligned}
E_{0} I_{0} \frac{\partial^{4} \widehat{y}_{0}}{\partial \widehat{x}^{4}}+\widehat{c} \frac{\partial \widehat{y}_{0}}{\partial \widehat{t}}+\rho_{0} A_{0} \frac{\partial^{2} \widehat{y}_{0}}{\partial \widehat{t}^{2}} \\
=\frac{\varepsilon_{0} b V_{0}^{2}}{2\left(\widehat{g}_{0}-\widehat{y}_{0}\right)^{2}}\left[1+0.65 \frac{\widehat{g}_{0}-\widehat{y}_{0}}{b}\right],
\end{aligned}
$$

where at the right-hand side is the electrostatic force including the fringe effect. The parameters $E_{0}, I_{0}, A_{0}, b, \widehat{c}, \rho_{0}$, $\widehat{y}_{0}, \widehat{t}$, and $\widehat{g}_{0}$ are Young's modulus, cross-section moment of inertia, cross-section area, beam width, damping per unit length, density, deflection, time, and the gap between cantilever and ground plate, respectively, and $\varepsilon_{0}=8.854 \times$ $10^{-12} \mathrm{C}^{2} \mathrm{~N}^{-1} \mathrm{~m}^{-2}$ is the permittivity of free space. In this work only uniform cantilevers are considered. However, dynamic modal characteristics for nonuniform beams can be found in $[28,29]$ in which self-adjoint differential equations and the method of factorization [30-32] have been used. The voltage $V_{0}$ considered in this paper is given by

$$
V_{0}=V_{0 D}+V_{0 A} \cos \widehat{\Omega}_{0} \widehat{t},
$$

where $V_{0 D}, V_{0 A}$, and $\widehat{\Omega}_{0}$ are the DC voltage, the amplitude of the AC voltage, and the frequency of the AC voltage, respectively. Dimensionless variables are introduced as follows:

$$
y_{0}=\frac{\widehat{y}_{0}}{\widehat{g}_{0}}, \quad x=\frac{\widehat{x}}{L}, \quad t=\widehat{t} \cdot L^{2} \sqrt{\frac{\rho_{0} A_{0}}{E_{0} I_{0}}},
$$

where $y_{0}, x, t$, and $L$ are the dimensionless beam displacement, dimensionless longitudinal coordinate, dimensionless time, and beam's length, respectively. Substituting (3) into (1), the dimensionless equation of motion results as follows:

$$
\frac{\partial^{2} y_{0}}{\partial t^{2}}+\frac{\partial^{4} y_{0}}{\partial x^{4}}=-c \frac{\partial y_{0}}{\partial t}+\frac{\delta_{0}}{\left(1-y_{0}\right)^{2}}+\frac{f \delta_{0}}{1-y_{0}}
$$


where $c$ is the dimensionless damping parameter and $f$ dimensionless fringe parameter

$$
c=\frac{\widehat{c} L^{2}}{\sqrt{\rho_{0} A_{0} E_{0} I_{0}}}, \quad f=\frac{0.65 \widehat{g}_{0}}{b} .
$$

The dimensionless voltage parameter $\delta_{0}$ is given by

$$
\delta_{0}=\left(\sqrt{\delta_{0 D}}+\sqrt{\delta_{0 A}} \cos \Omega_{0} t\right)^{2}
$$

where

$$
\begin{gathered}
\delta_{0 D}=\frac{\varepsilon_{0} b L^{4} V_{0 D}^{2}}{2 E_{0} I_{0} \widehat{g}_{0}^{3}}, \quad \delta_{0 A}=\frac{\varepsilon_{0} b L^{4} V_{0 A}^{2}}{2 E_{0} I_{0} \widehat{g}_{0}^{3}}, \\
\Omega_{0}=\widehat{\Omega}_{0} \cdot L^{2} \sqrt{\frac{\rho_{0} A_{0}}{E_{0} I_{0}}} .
\end{gathered}
$$

$\delta_{0 D}$ is the dimensionless DC voltage parameter, $\delta_{0 A}$ is the dimensionless AC voltage parameter, and $\Omega_{0}$ is the dimensionless frequency of excitation.

\section{Method of Multiple Scales}

The system under consideration is weakly damped and softly actuated; that is, the dimensionless parameters from (5) and (6) are small (less than or equal to 0.1). In this case the method of multiple scales (MMS) can be used to investigate the behavior of the system. A small bookkeeping parameter $\varepsilon$ is then introduced in (4) as follows:

$$
\frac{\partial^{2} y_{0}}{\partial t^{2}}+\frac{\partial^{4} y_{0}}{\partial x^{4}}=-\varepsilon c \frac{\partial y_{0}}{\partial t}+\varepsilon \frac{\delta_{0}}{\left(1-y_{0}\right)^{2}}+\varepsilon \frac{f \delta_{0}}{1-y_{0}}
$$

The last two terms at the right hand side of (8) are expanded in Taylor series and the first terms up to the cubic power are retained. MMS is a perturbation method; therefore "the first few steps reveal the important features of the solution and the remaining ones give small corrections"; that is, "one may calculate just few terms in a perturbation series" [33]. Throughout this paper we only discuss a first-order uniform expansion of the solution [18-21,34-41]. Thus, an approximate solution to this weakly nonlinear system in the form of a first-order uniformly valid expansion is sought:

$$
y_{0}\left(T_{0}, T_{1}, x, \varepsilon\right)=u_{0}\left(T_{0}, T_{1}, x\right)+\varepsilon u_{1}\left(T_{0}, T_{1}, x\right),
$$

where $T_{0}, T_{1}$ are the fast and the slow time scales defined as $T_{0}=t$ and $T_{1}=\varepsilon t$, respectively. The derivative with respect to time can be expressed in terms of the derivatives with respect to the two time scales as $\partial / \partial t=D_{0}+\varepsilon D_{1}$, where $D_{0}=\partial / \partial T_{0}$ and $D_{1}=\partial / \partial T_{1}$. Using (9) and abovementioned time derivatives into (8) after Taylor expansion and equating terms of like powers of $\varepsilon$, two problems result as follows:

$$
\begin{aligned}
& \varepsilon^{0}: D_{0}^{2} u_{0}+u_{0}^{(4)}=0 \\
& \varepsilon^{1}: D_{0}^{2} u_{1}+u_{1}^{(4)}=-2 D_{0} D_{1} u_{0}-c D_{0} u_{0} \\
& +\delta_{0}\left[1+2 u_{0}+3 u_{0}^{2}+4 u_{0}^{3}\right] \\
& +f \delta_{0}\left[1+u_{0}+u_{0}^{2}+u_{0}^{3}\right] .
\end{aligned}
$$

The solution of the boundary value problem for cantilevers associated to (10) is given by

$$
u_{0}=\phi_{k}(x)\left[A_{k}\left(T_{1}\right) e^{i \omega_{k} T_{0}}+\bar{A}_{k}\left(T_{1}\right) e^{-i \omega_{k} T_{0}}\right] \text {, }
$$

where coefficients $A_{k}$ and $\bar{A}_{k}$ are to be determined, and $\omega_{k}$ and $\phi_{k}$ are natural frequencies and their corresponding mode shapes, respectively.

\section{Nonlinear Parametric Resonance}

Next, (12) is substituted into (11). The nonlinear parametric resonance for AC frequency near natural frequency is investigated. The nearness of the AC voltage frequency $\Omega_{0}$ to the natural frequency $\omega_{k}$ is given by

$$
\Omega_{0}=\omega_{k}+\varepsilon \sigma,
$$

where $\sigma$ is a detuning parameter representing frequency offset. The secular terms are collected from the resulting equation and set equal to zero:

$$
\begin{gathered}
-2 i \omega_{k} \varphi_{k} A_{k}^{\prime}-i c \omega_{k} \varphi_{k} A_{k}+(1+f) \sqrt{\delta_{0 D} \delta_{0 A}} e^{i \sigma T_{1}} \\
+(2+f) \varphi_{k}\left[\left(\delta_{0 D}+\frac{\delta_{0 A}}{2}\right) A_{k}+\frac{\delta_{0 A}}{4} \bar{A}_{k} e^{i 2 \sigma T_{1}}\right] \\
+(3+f) \varphi_{k}^{2} \sqrt{\delta_{0 D} \delta_{0 A}}\left(A_{k}^{2} e^{-i \sigma T_{1}}+2 A_{k} \bar{A}_{k} e^{i \sigma T_{1}}\right) \\
+(4+f) \varphi_{k}^{3}\left[\left(\delta_{0 D}+\frac{\delta_{0 A}}{2}\right) 3 A_{k}^{2} \bar{A}_{k}\right. \\
\left.+\frac{\delta_{0 A}}{4}\left(A_{k}^{3} e^{-2 i \sigma T_{1}}+3 A_{k} \bar{A}_{k}^{2} e^{2 i \sigma T_{1}}\right)\right]=0 .
\end{gathered}
$$

Consider the following $g$ coefficients in the case of uniform cantilevers [21]:

$$
g_{n k k}=\left\langle\varphi_{k}^{n}, \varphi_{k}\right\rangle=\int_{0}^{1} \varphi_{k}^{n} \varphi_{k} d x
$$


The amplitude $A_{k}$ is considered as follows:

$$
A_{k}=\frac{1}{2} a_{k} e^{i \beta_{k}}
$$

where $a_{k}$ and $\beta_{k}$ are the real amplitude and phase of the EAMEMS-CR. Substituting (15) and (16) into (14) and separating the real and the imaginary parts, one obtains the slow scale system of differential equations given by

$$
\begin{aligned}
a_{k}^{\prime}= & -\frac{c}{2} a_{k}+\frac{2 C_{1}}{\omega_{k} g_{1 k k}} \sqrt{\delta_{0 D} \delta_{0 A}} \sin \gamma_{k}+\frac{C_{2}}{\omega_{k}} \frac{\delta_{0 A} a_{k}}{4} \sin 2 \gamma_{k} \\
& +\frac{C_{3}}{\omega_{k}} \frac{g_{2 k k}}{g_{1 k k}} \sqrt{\delta_{0 D} \delta_{0 A}} \frac{a_{k}^{2}}{2} \sin \gamma_{k}+\frac{C_{4}}{\omega_{k}} \frac{g_{3 k k}}{g_{1 k k}} \frac{\delta_{0 A} a_{k}^{3}}{8} \sin 2 \gamma_{k}, \\
a_{k} \gamma_{k}^{\prime}= & a_{k} \sigma+\frac{2 C_{1}}{\omega_{k} g_{1 k k}} \sqrt{\delta_{0 D} \delta_{0 A}} \cos \gamma_{k} \\
& +\frac{C_{2}}{\omega_{k}}\left[\left(\delta_{0 D}+\frac{\delta_{0 A}}{2}\right) a_{k}+\frac{\delta_{0 A}}{4} a_{k} \cos 2 \gamma_{k}\right] \\
& +\frac{2 \alpha}{\omega_{k}} a_{k}+\frac{C_{3}}{\omega_{k}} \frac{g_{2 k k}}{g_{1 k k}} \sqrt{\delta_{0 D} \delta_{0 A}} \frac{3 a_{k}^{2}}{2} \cos \gamma_{k} \\
& +\frac{C_{4}}{\omega_{k}} \frac{g_{3 k k}}{g_{1 k k}}\left[\left(\delta_{0 D}+\frac{\delta_{0 A}}{2}\right) \frac{3}{4} a_{k}^{3}+\frac{\delta_{0 A}}{4} a_{k}^{3} \cos 2 \gamma_{k}\right] \\
& +\frac{15 \alpha}{2 \omega_{k}} \frac{g_{3 k k}}{g_{1 k k}} a_{k}^{3},
\end{aligned}
$$

where

$$
\begin{gathered}
\gamma_{k}=\sigma T_{1}-\beta_{k}, \\
C_{n}=\frac{n+f}{2} .
\end{gathered}
$$

Therefore the steady state solutions $\left(a_{k}^{\prime}=0, \gamma_{k}^{\prime}=0\right)$ are as follows:

$$
\begin{aligned}
&- \frac{\left(2 C_{1}+C_{3} g_{2 k k}\left(a_{k}^{2} / 2\right)\right) \sqrt{\delta_{0 D} \delta_{0 A}} \sin \gamma_{k}}{a_{k} \omega_{k} g_{1 k k}} \\
&=-\frac{c}{2}+\left[C_{2}+\frac{C_{4}}{2} \frac{g_{3 k k}}{g_{1 k k}} a_{k}^{2}\right] \delta_{0 A} \frac{\sin 2 \gamma_{k}}{4 \omega_{k}}, \\
& \sigma=-\frac{1}{a_{k} \omega_{k} g_{1 k k}}\left(2 C_{1}+\frac{3}{2} C_{3} g_{2 k k} a_{k}^{2}\right) \sqrt{\delta_{0 D} \delta_{0 A}} \cos \gamma_{k} \\
&-\left(C_{2}+C_{4} \frac{g_{3 k k}}{g_{1 k k}} a_{k}^{2}\right) \frac{\delta_{0 A}}{4 \omega_{k}} a_{k} \cos 2 \gamma_{k}-\frac{1}{\omega_{k}}\left(\delta_{0 D}+\frac{\delta_{0 A}}{2}\right) \\
& \quad \times\left(C_{2}+\frac{3}{4} C_{4} \frac{g_{3 k k}}{g_{1 k k}} a_{k}^{2}\right)-\frac{\alpha}{\omega_{k}}\left(2+\frac{15}{2} a_{k}^{2} \frac{g_{3 k k}}{g_{1 k k}}\right) .
\end{aligned}
$$

\section{Reduced Order Model Method}

In order to validate the results from MMS, (20) and (21), a different method, namely, ROM, is used. The ROM yields a set of numerically solvable ordinary differential equations. It becomes more accurate with the increase of number of terms in the model. ROM provides only stable steady-state solutions. Below is the derivation of the ROM equations used in the numerical simulation. Equation (4) is multiplied by $\left(1-y_{0}\right)^{2}$; therefore no term will have denominator:

$$
\begin{aligned}
& \left(1-y_{0}\right)^{2} \frac{\partial^{2} y_{0}}{\partial t^{2}}+\left(1-y_{0}\right)^{2} \frac{\partial^{4} y_{0}}{\partial x^{4}} \\
& =-c\left(1-y_{0}\right)^{2} \frac{\partial y_{0}}{\partial \hat{t}}+\left(1-y_{0}\right) f \delta_{0}+\delta_{0} .
\end{aligned}
$$

Next, a two-term (2T) ROM is used

$$
y_{0}=u_{01}(t) * \phi_{1}(x)+u_{02}(t) * \phi_{2}(x)
$$

where $u_{01}$ and $u_{02}$ are functions of time to be found through integration and

$$
\begin{aligned}
\phi_{n}=-[ & \cos \left(\sqrt{\omega_{n}} \cdot x\right)-\cosh \left(\sqrt{\omega_{n}} \cdot x\right) \\
& \left.+C_{n}\left(\sin \left(\sqrt{\omega_{n}} \cdot x\right)-\sinh \left(\sqrt{\omega_{n}} \cdot x\right)\right)\right]
\end{aligned}
$$

are the mode shape functions of the beam. In this case $n=1$, 2; that is, only two-term (2T) ROM is considered. Constants $\omega_{n}$ are the dimensionless natural frequencies of uniform cantilevers and $C_{n}$ are the coefficients of the corresponding mode shapes equation (24), all given in $[18,19,27]$. The following relationship holds since $\omega_{n}$ and $\phi_{n}$ are natural frequencies and mode shapes:

$$
\frac{\partial^{4} y_{0}}{\partial x^{4}}=\omega_{1}^{2} u_{01} \phi_{1}+\omega_{2}^{2} u_{02} \phi_{2} .
$$

Substituting (23)-(25) into (22), multiplying the resulting equation by $\phi_{1}$, and then integrating from 0 to 1 gives the first ROM second-order differential equation. The second ROM second-order differential equation is obtained similarly by multiplying by $\phi_{2}$ and then integrating from 0 to 1 . The two resulting equations are then integrated.

\section{Numerical Results}

Numerical simulations using Matlab are conducted for parametric resonance of MEMS cantilever resonators under soft actuation (i.e, actuation leading to small to very small amplitudes relative to the gap when the AC frequency is away from resonance and/or small to very small deflections if DC) and weak damping. Both DC and AC voltage components between the EA-MEMS-CR and the ground plate are considered. The physical characteristics of EAMEMS-CRs considered for numerical simulations are given in Table 1. From the values listed in Table 1, the dimensionless parameters can be calculated for each case, as listed in Table 2. First, consider the frequency response of EA-MEMS-CRs 


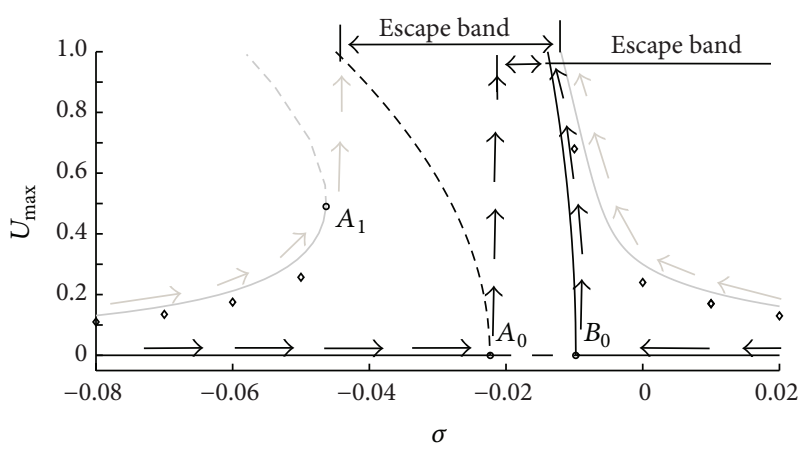

(a)

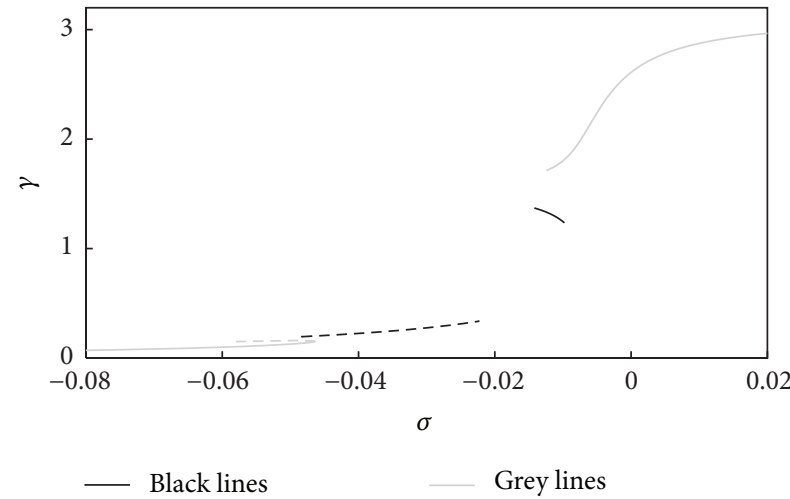

(b)

FIGURE 2: Frequency response of EA-MEMS-CR. AC frequency is near natural frequency. (a) Amplitude-frequency response and (b) phase-frequency response. $U_{\max }$ is the dimensionless amplitude of the free end, $\sigma$ dimensionless detuning frequency equation (13), and $\gamma$ dimensionless phase equation (18). Zero value for $\sigma$ corresponds to natural frequency. Two methods are used, namely, MMS and twoterm (2T) ROM. In both figures (a) and (b) two cases are shown: (i) soft AC with zero DC bias (black lines: MMS $\delta_{0 D}=0.0$ ) and (ii) soft AC with nonzero DC bias (grey lines: MMS $\delta_{0 D}=0.001 ; \diamond 2 \mathrm{~T}-\mathrm{ROM}$ $\left.\delta_{0 D}=0.001\right)$. The AC voltage, fringe, and damping are $\delta_{0 A}=0.1, f=$ 0.26 , and $c=0.01$, respectively.

under AC soft voltage of frequency near natural frequency of the cantilever and zero DC bias. The frequency response is given by (20) and (21), and the case of $\delta_{0 A}=0.1$ and $\delta_{0 D}=0$ is illustrated in Figure 2. These results are in agreement with data reported in the literature [26, 27]. Second, if both soft AC voltage $\delta_{0 A}=0.1$ of frequency near natural frequency and soft DC bias $\delta_{0 D}=0.001$, then the frequency response of EA-MEMS-CRs is significantly altered, as shown in Figure 2. The DC voltage parameter $\delta_{0 D}$ is $1 \%$ of the $\mathrm{AC}$ excitation voltage. Figure 2(a) shows the amplitude-frequency response of the resonator, where the frequency is given by the detuning parameter $\sigma$, (13), and the amplitude $U_{\max }$ is the dimensionless vibration amplitude of the cantilever tip with respect to the initial gap distance between the cantilever and ground plate. A value of zero of $U_{\max }$ means the cantilever has no motion, a value of 0.5 means its amplitude is half the gap, and values of one or greater than one mean pull-in phenomenon (contact with the ground plate). Figure 2(b) shows the phase-frequency response of
TABLE 1: Physical characteristics of a typical microbeam.

\begin{tabular}{lcc}
\hline Parameter & Symbol & Value \\
\hline Beam width & $b$ & $20 \mu \mathrm{m}$ \\
Beam length & $L$ & $300 \mu \mathrm{m}$ \\
Beam thickness & $h$ & $2.0 \mu \mathrm{m}$ \\
Initial gap distance & $\widehat{g}_{0}$ & $8.0 \mu \mathrm{m}$ \\
Material density & $\rho_{0}$ & $2330 \mathrm{~kg} / \mathrm{m}^{3}$ \\
Young's modulus & $E_{0}$ & $169 \mathrm{GPa}$ \\
Quality factor & $Q$ & 350 \\
Peak AC voltage & $V_{0 A}$ & $12.5 \mathrm{~V}$ \\
Peak DC voltage & $V_{0 D}$ & $1.25 \mathrm{~V}$ \\
\hline
\end{tabular}

TABLE 2: Dimensionless parameters.

\begin{tabular}{lcc}
\hline Parameter & Symbol & Value \\
\hline Damping coefficient & $c$ & 0.01 \\
Voltage amplitude coefficient & $\delta_{0 D}$ & 0.001 \\
Voltage amplitude coefficient & $\delta_{0 A}$ & 0.1 \\
Fringe coefficient & $f$ & 0.26 \\
\hline
\end{tabular}

the resonator, where the phase is given by $\gamma$. Hereafter solid and dash lines represent stable and unstable steady-state solutions, respectively. In what follows a comparison between the two cases of AC voltage with zero DC bias and AC voltage with nonzero DC bias is conducted.

(1) If soft AC voltage with zero DC bias is applied (i.e., $\left.\delta_{0 A}=0.1, \delta_{0 D}=0\right)$, the amplitude-frequency response $\left(\sigma-U_{\max }\right)$ consists of two Hopf bifurcations, subcritical and supercritical as illustrated in Figure 2(a). The subcritical Hopf bifurcation with $A_{0}$ bifurcation point consists of (a) stable zero steady-state amplitudes (solid line) at the left-hand side of $A_{0}$, (b) unstable zero steady-state amplitudes (dash line) at the right-hand side of $A_{0}$, and (c) unstable nonzero steadystate amplitudes (dash line) branching up from $A_{0}$. The supercritical Hopf bifurcation with $B_{0}$ as bifurcation point consists of (a) unstable zero steady-state amplitudes (dash line) at the left-hand side of $B_{0}$, (b) stable zero steady-state amplitudes (solid line) at the right-hand side of $B_{0}$, and (c) stable nonzero steady-state amplitudes (solid line) branching up from $B_{0}$.

As the frequency is swept up the steady-state amplitude $U_{\max }$ of the tip of the cantilever Figure 2(a) remains zero until the bifurcation point $A_{0}$ of the subcritical Hopf bifurcation is reached. At this $A_{0}$ bifurcation frequency EA-MEMSCR loses stability and undergoes a pull-in phenomenon (contact with the ground plate) reaching a value of one for the dimensionless amplitude $U_{\max }$; that is, the dimensional amplitude reaches the gap value. This process is illustrated by the black arrows from the origin to $A_{0}$ and then straight up. As the frequency is swept down the steady-state amplitude Figure 2(a) remains zero until the bifurcation point $B_{0}$ of the supercritical Hopf bifurcation is reached. As the frequency continues to be swept down from $B_{0}$, the amplitude continuously increases until pull-in occurs. This is illustrated by the black arrows from high frequencies to $B_{0}$ and then up. 
The phase-frequency response $\sigma-\gamma$ shows only a phase around $\pi / 2$ for the stable branch from $B_{0}$ of nonzero amplitudes, as shown in Figure 2(b).

(2) If soft AC voltage with soft DC bias $\left(\delta_{0 A}=0.1, \delta_{0 D}=\right.$ 0.001 ), the amplitude-frequency response consists of a cyclic fold bifurcation in lower frequencies and no bifurcation branch in higher frequencies as shown in Figure 2(a). The cyclic fold bifurcation with $A_{1}$ bifurcation point consists of (a) stable nonzero steady-state amplitudes (solid line) below $A_{1}$ and (b) unstable nonzero steady-state amplitudes (dash line) above $A_{1}$. The no bifurcation branch consists of nonzero steady-state amplitudes above the supercritical $B_{0}$ Hopf bifurcation.

As the frequency is swept up the steady-state amplitude $U_{\max }$ of the tip of the cantilever Figure 2(a) increases continuously until it reaches the bifurcation point $A_{1}$ of the cyclic fold bifurcation. The $A_{1}$ bifurcation amplitude is about half the gap in this case. At this $A_{1}$ bifurcation frequency the EA-MEMS-CR loses stability and undergoes a pull-in phenomenon. This process is illustrated by the grey arrows from lower frequencies to $A_{1}$ and then straight up. As the frequency is swept down, the steady-state amplitude in Figure 2(a) increases continuously until pull-in occurs. This is illustrated by the grey arrows along the branch above $B_{0}$.

ROM points in Figure 2, which are represented by diamonds, show a good agreement with MMS.

\section{Discussion and Conclusions}

Figure 2 shows the influence of soft DC bias on the frequency response of EA-MEMS-CRs. The soft DC bias of the soft AC voltage near natural frequency changes the subcritical Hopf bifurcation, with $A_{0}$ bifurcation point, into a cyclic fold bifurcation, with $A_{1}$ bifurcation point, as shown in Figure 2(a). As one can notice the DC bias shifts the bifurcation frequency to lower values $\left(\sigma_{A_{1}}<\sigma_{A_{0}}\right)$ and the bifurcation dimensionless amplitude (when the resonator loses stability) from zero at $A_{0}$ to about 0.5 of the gap at $A_{1}$. The escape frequency band for $\delta_{0 D}=0$ is enlarged significantly for $\delta_{0 D}=0.001$ (about three times in this case) as shown in Figure 2(a). Within the escape band the resonator goes into pull-in regardless of the initial amplitude. For all stable steady-state amplitudes of the cyclic fold bifurcation ( $A_{1}$ bifurcation point) the phase is almost zero.

Also, the soft DC bias has a significant influence on the frequency-phase response as shown in Figure 2(b). As the frequency is swept down the phase decreases from $\pi$ to about $\pi / 2$ if the soft DC bias is not zero $\delta_{0 D} \neq 0$, while the phase for soft AC with zero DC bias, $\delta_{0 D}=0$, increases below $\pi / 2$ for a small range of frequencies corresponding to nonzero amplitudes of the supercritical Hopf bifurcation.

Figure 3 shows the continuous transition of the frequency response from soft $\mathrm{AC}$ voltage with zero $\mathrm{DC}$ bias $\delta_{0 D}=$ 0 to soft $\mathrm{AC}$ with $\mathrm{DC}$ bias $\delta_{0 D} \neq 0$, that is, the continuous transition from subcritical Hopf bifurcation and supercritical Hopf bifurcation to cyclic fold bifurcation and no bifurcation branch, respectively. The nonzero DC voltage values considered in this figure are $0.02 \%, 0.05 \%$, and $0.1 \%$ of the $\mathrm{AC}$ voltage.

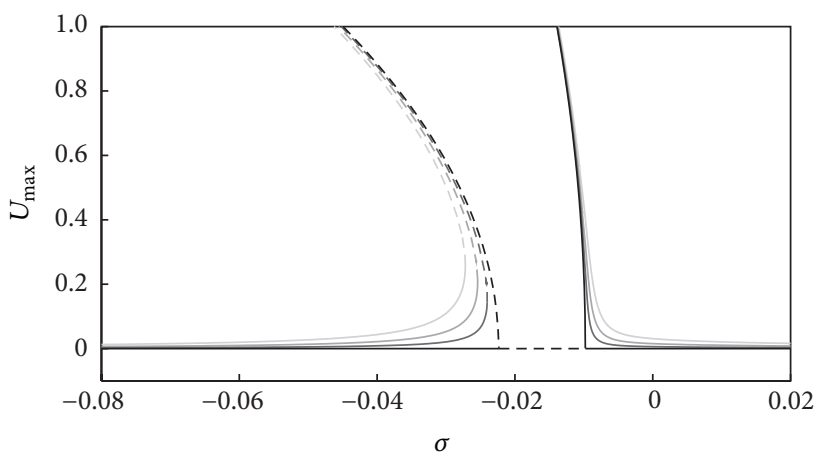

(a)

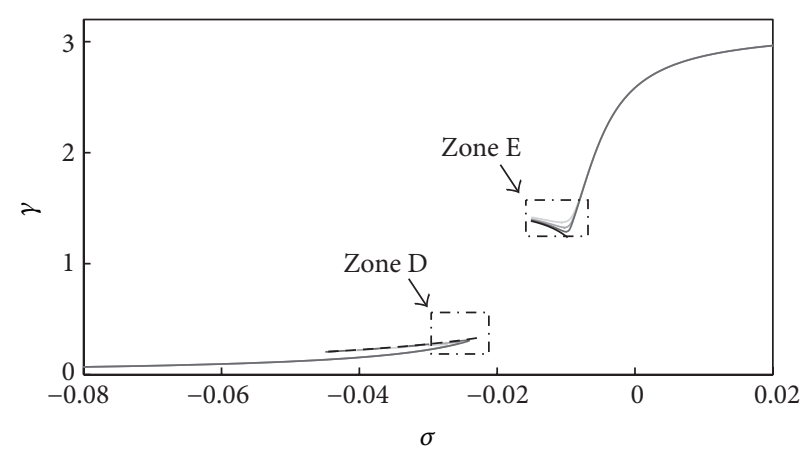

(b)

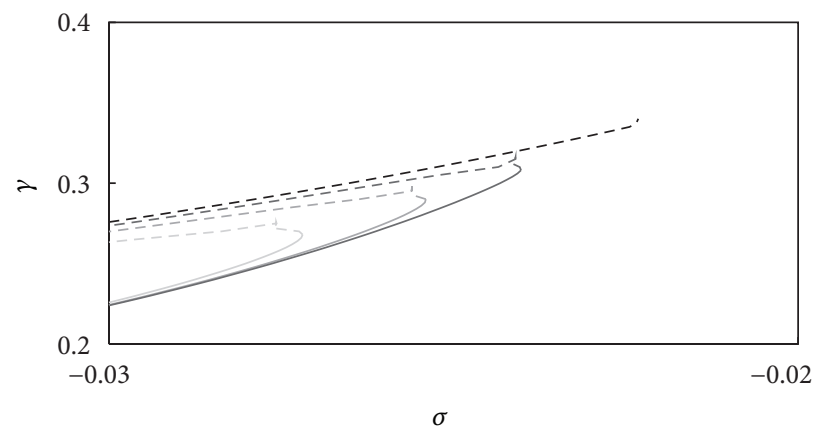

(c)

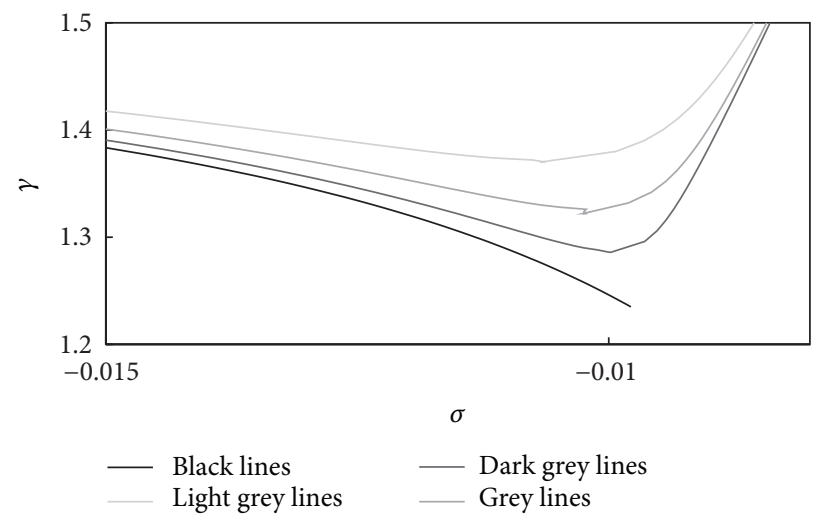

(d)

Figure 3: Continuous transition of the frequency response from Hopf bifurcation to cyclic fold bifurcation due to DC bias $\delta_{0 D}$. $\mathrm{AC}$ frequency is near natural frequency. (a) Amplitude-frequency response, (b) phase-frequency response, (c) Zone D detail, and (d) Zone E detail. MMS is used. Four cases are shown in all figures: (i) zero DC bias (black lines): $\delta_{0 D}=0.0$, (ii) dark grey lines: $\delta_{0 D}=$ 0.00002 , (iii) grey lines: $\delta_{0 D}=0.00005$, and (iv) light grey lines: $\delta_{0 D}$ $=0.0001$. The AC voltage, fringe, and damping are $\delta_{0 A}=0.1, f=0.26$, and $c=0.01$, respectively. 


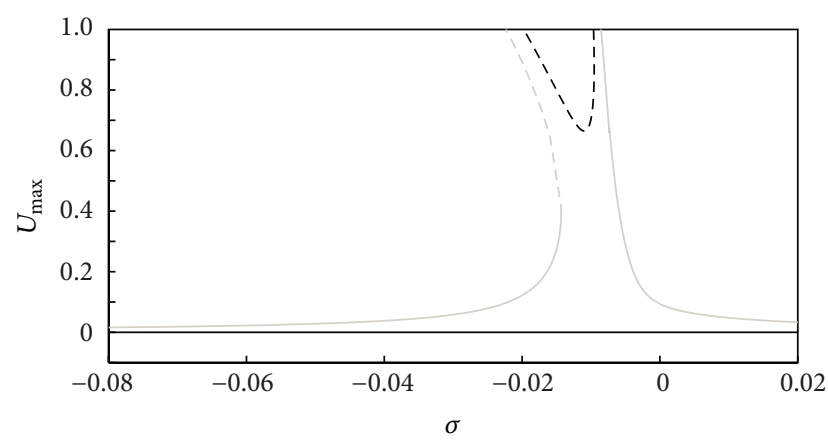

(a)

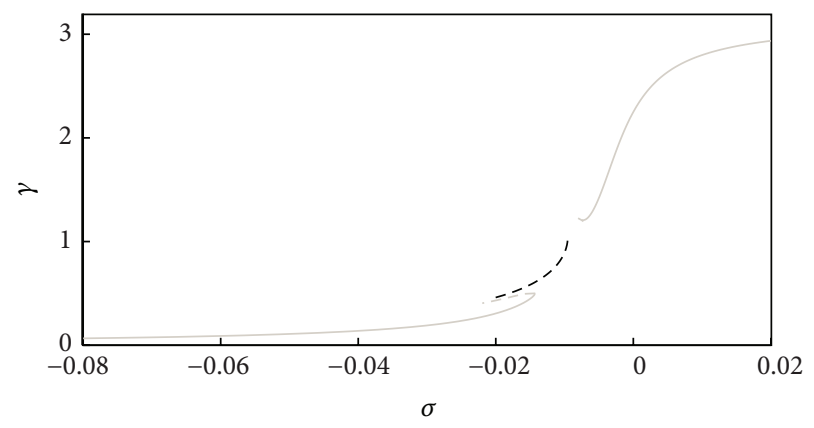

— Black lines _ Grey line

(b)

FIgURE 4: Creation of escape band due to DC bias. AC frequency is near natural frequency. (a) Amplitude-frequency response and (b) phase-frequency response. MMS is used. AC voltage $\delta_{0 A}$ is relatively small (in this case half of the one used in Figures 2 and 3). In each figure two cases are shown: (i) zero DC bias (black lines): $\delta_{0 D}=0.0$ and there is no escape band and (ii) nonzero DC bias (grey lines): $\delta_{0 D}=0.0003$ and an escape band is created. The AC voltage, fringe, and damping are $\delta_{0 A}=0.05, f=0.26$, and $c=0.01$, respectively.

Figure 4 shows another interesting and significant influence of the soft DC voltage on the frequency response of EA-MEMS-CRs, namely, the creation of escape band. In the case of softer AC voltage, such as half the one in Figures 2 and 3 , and zero DC bias, the amplitude-frequency response in Figure 4(a) consists of unstable (dash black line) solutions $\left(\delta_{0 A}=0.05, \delta_{0 D}=0\right)$ in the range of amplitudes of 0.6 to 1 , and stable zero amplitude horizontal branch $\left(U_{\max }=0\right.$ for any $\sigma$ ). One can notice that nonzero steady-state amplitudes or pull-in cannot be reached from small $\left(U_{\max }<0.6\right)$ initial amplitudes. For small initial amplitudes $\left(U_{\max }<0.6\right)$ the system settles to zero amplitude, that is, to a point on the stable zero amplitude horizontal branch. Also the range of frequencies corresponding to stable nonzero amplitudes is very narrow. As the DC bias becomes nonzero $\left(\delta_{0 A}=0.05\right.$, $\left.\delta_{0 D}=0.0003\right)$, the nonzero amplitudes and zero amplitude branches coalesce into a cyclic fold bifurcation (left-hand side) and a no bifurcation branch of stable steady-state solutions (right-hand side). Nonzero steady-state amplitudes or pull-in can now be reached from small initial amplitudes. For instance amplitudes greater than 0.15 of the gap or pull-in can be reached for frequencies $\sigma$ between about -0.015 and

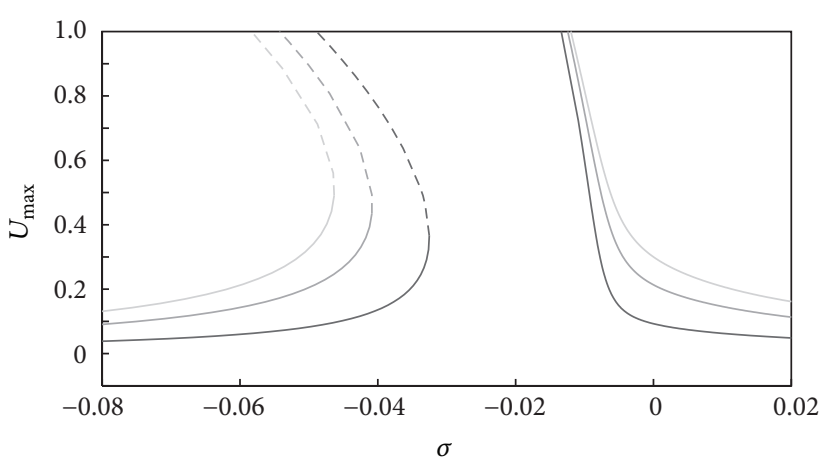

(a)

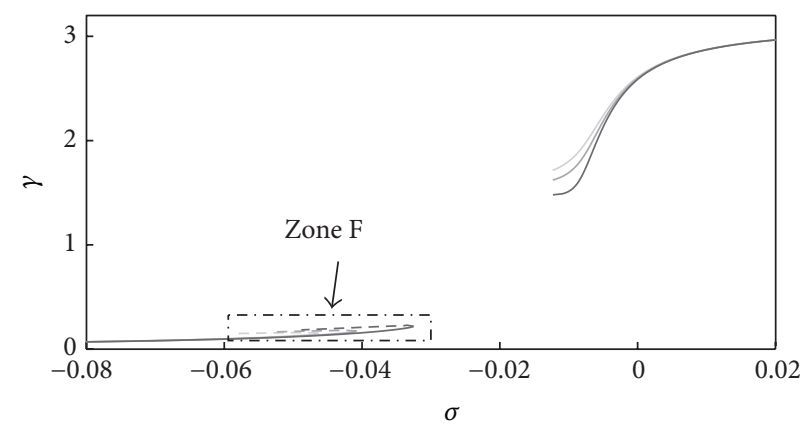

(b)

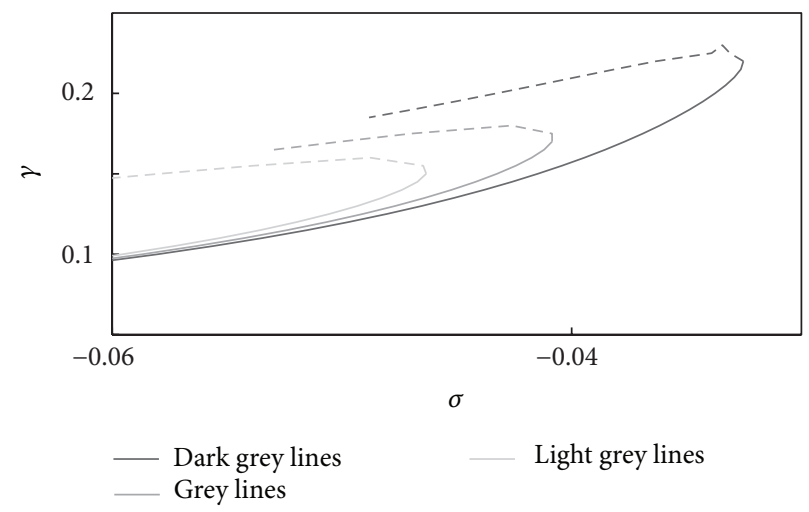

(c)

FIGURE 5: DC bias effect on the frequency response. AC frequency is near natural frequency. (a) Amplitude-frequency response, (b) phase-frequency response, and (c) Zone F detail. MMS is used. Three cases of DC bias are shown in all figures: (i) black lines: $\delta_{0 D}$ $=0.0003$, (ii) dark grey lines: $\delta_{0 D}=0.0007$, and (iii) light grey lines: $\delta_{0 D}=0.001$. The AC voltage, fringe, and damping are $\delta_{0 A}=0.1, f=$ 0.26 , and $c=0.01$, respectively.

-0.005 . Similarly in the frequency-phase response, the one branch for $\delta_{0 D}=0$ splits into two branches for $\delta_{0 D}=0.0003$.

Figure 5 shows the effect of the soft DC bias on the frequency response of the resonator. As the DC bias increases, the bifurcation point (similar to $A_{1}$ in Figure 2) shifts continuously to lower frequencies and larger amplitudes, and the right-hand side branch of stable steady-state solutions shifts to larger amplitudes, as shown in Figure 5(a). Therefore, increasing the DC bias widens the window of frequencies for which the system reaches pull-in from any initial amplitude. 
Also, the range of frequencies for which nonzero stable steady-state amplitudes can be reached increases as well. In the frequency-phase response in Figure 5(b), the increase of the DC voltage results in a slight increase of the phase of the stable steady-state solutions.

In conclusion, the response of electrostatically actuated system composed of a ground plate and a parallel microcantilever resonator excited by different combinations of DC bias and AC voltage of frequencies near natural frequency of EAMEMS-CR has been investigated. This actuation resulted in a nonlinear parametric resonance. The differential equation of motion has been nondimensionalized and MMS has been used to investigate the case of soft actuation and soft damping. Isolating secular terms enabled the description of the steadystate solution of the near natural frequency case. Moreover ROM method has been used, and an agreement with MMS resulted. Numerical simulations showed that, for soft AC voltage and zero DC bias, the results are in agreement with data reported in the literature [26, 27]. The addition of soft DC bias changed the nature of the bifurcation from subcritical Hopf to cyclic fold and supercritical Hopf to no bifurcation nonzero steady-state amplitude branch. Also, it resulted in a larger escape band; that is, range of frequencies for which pull-in occurs regardless the value of the initial amplitude. Moreover, the amplitude at which the resonator loses stability ranged from zero to 0.5 for the numerical simulations conducted. The DC bias eliminated stable zero steady-states amplitudes and shifted the bifurcation point to lower frequencies and larger amplitudes. It also caused the phase response of the right-hand stable branch to be greater than $\pi / 2$ as shown in Figure 5. A continuous transition of the frequency response from AC with zero DC bias to AC with DC bias has been reported in Figure 3.

One application of this work is finding the level of unwanted DC bias in a system. This level can be found by determining the amplitude and frequency of the cyclic fold bifurcation point $A_{1}$ in Figure 2. The lower the DC bias, the lower the bifurcation amplitude and the larger the bifurcation frequency. However this bifurcation frequency is less than the Hopf bifurcation frequency of point $A_{0}$. Another application of this work consists of controlling the size of the escape band through the DC bias. Larger value of DC bias results in a larger escape band.

Limitations of this work are as follows. (1) The results are valid for weakly nonlinearities and small to moderate amplitudes. Therefore the MMS fails to predict the pullin instability at large amplitudes as the frequency is swept down. It has been reported in the literature that a reduced order model (ROM) of five terms predicts this pull-in instability [18-21]. (2) The pull-in AC and DC voltages are not predicted, since this work is concerned only with frequency response. Future investigation regarding the voltage amplitude response is to be conducted.

\section{Disclosure}

University of Texas Pan American becomes University of Texas-Rio Grande Valley starting fall 2015.

\section{Conflict of Interests}

The authors declare that there is no conflict of interests regarding the publication of this paper.

\section{Acknowledgments}

This material is based on research sponsored by Air Force Research Laboratory under Agreement no. FA8650-07-25061. The U.S. Government is authorized to reproduce and distribute reprints for governmental purposes notwithstanding any copyright notation thereon. The views and conclusions contained herein are those of the authors and should not be interpreted as necessarily representing the official policies or endorsements, either expressed or implied, of Air Force Research Laboratory or the U.S. Government.

\section{References}

[1] M. I. Younis and F. Alsaleem, "Exploration of new concepts for mass detection in electrostatically-actuated structures based on nonlinear phenomena," Journal of Computational and Nonlinear Dynamics, vol. 4, no. 2, Article ID 021010, 15 pages, 2009.

[2] J. H. Zhao, S. J. Yu, K. Li, Y. Dong, Q. Zhou, and Z. You, "Improvement of a micro-beam in a smart gas sensor with resonating mechanism," in Proceedings of the 4th IEEE International Conference on Nano/Micro Engineered and Molecular Systems (NEMS '09), pp. 371-375, January 2009.

[3] F. M. Alsaleem, M. I. Younis, and H. M. Ouakad, "On the nonlinear resonances and dynamic pull-in of electrostatically actuated resonators," Journal of Micromechanics and Microengineering, vol. 19, no. 4, Article ID 045013, 2009.

[4] J. Teva, G. Abadal, Z. J. Davis et al., "On the electromechanical modelling of a resonating nano-cantilever-based transducer," Ultramicroscopy, vol. 100, no. 3-4, pp. 225-232, 2004.

[5] M. Rasekh and S. E. Khadem, "Pull-in analysis of an electrostatically actuated nano-cantilever beam with nonlinearity in curvature and inertia," International Journal of Mechanical Sciences, vol. 53, no. 2, pp. 108-115, 2011.

[6] R. C. Batra, M. Porfiri, and D. Spinello, "Electromechanical model of electrically actuated narrow microbeams," Journal of Microelectromechanical Systems, vol. 15, no. 5, pp. 1175-1189, 2006.

[7] S. Chaterjee and G. Pohit, "A large deflection model for the pullin analysis of electrostatically actuated microcantilever beams," Journal of Sound and Vibration, vol. 322, no. 4-5, pp. 969-986, 2009.

[8] N. Kacem, S. Baguet, S. Hentz, and R. Dufour, "Computational and quasi-analytical models for non-linear vibrations of resonant MEMS and NEMS sensors," International Journal of NonLinear Mechanics, vol. 46, no. 3, pp. 532-542, 2011.

[9] Z.-Q. Guan, G. Luo, L. Montelius, and H.-X. Xu, "Electromechanical behavior of interdigitated $\mathrm{SiO} 2$ cantilever arrays," Chinese Physics Letters, vol. 27, no. 2, Article ID 028503, 2010.

[10] S. K. De and N. R. Aluru, "Full-Lagrangian schemes for dynamic analysis of electrostatic MEMS," Journal of Microelectromechanical Systems, vol. 13, no. 5, pp. 737-758, 2004.

[11] H. Takamatsu and T. Sugiura, "Nonlinear vibration of electrostatic MEMS under DC and AC applied voltage," in Proceedings of the 2005 International Conference on MEMS, NANO and Smart Systems (ICMENS '05), pp. 423-424, July 2006. 
[12] W.-M. Zhang, G. Meng, and K.-X. Wei, "Dynamics of nonlinear coupled electrostatic micromechanical resonators under twofrequency parametric and external excitations," Shock and Vibration, vol. 17, no. 6, pp. 759-770, 2010.

[13] Y. C. Hu, C. M. Chang, and S. C. Huang, "Some design considerations on the electrostatically actuated microstructures," Sensors and Actuators A: Physical, vol. 112, no. 1, pp. 155-161, 2004.

[14] L. Jiang, M. Hassan, R. Cheung et al., "Dry release fabrication and testing of SiC electrostatic cantilever actuators," Microelectronic Engineering, vol. 78-79, no. 1-4, pp. 106-111, 2005.

[15] A. Hajnayeb and S. E. Khadem, "Nonlinear vibration and stability analysis of a double-walled carbon nanotube under electrostatic actuation," Journal of Sound and Vibration, vol. 331, no. 10, pp. 2443-2456, 2012.

[16] A. M. Elshurafa, K. Khirallah, H. H. Tawfik, A. Emira, A. K. S. Abdel Aziz, and S. M. Sedky, "Nonlinear dynamics of spring softening and hardening in folded-mems comb drive resonators," Journal of Microelectromechanical Systems, vol. 20, no. 4, Article ID 5772897, pp. 943-958, 2011.

[17] M. H. Ghayesh, H. Farokhi, and M. Amabili, "Nonlinear behaviour of electrically actuated MEMS resonators," International Journal of Engineering Science, vol. 71, pp. 137-155, 2013.

[18] D. I. Caruntu, I. Martinez, and K. N. Taylor, "Voltage-amplitude response of alternating current near half natural frequency electrostatically actuated MEMS resonators," Mechanics Research Communications, vol. 52, pp. 25-31, 2013.

[19] D. I. Caruntu, I. Martinez, and M. W. Knecht, "ROM analysis of frequency response of AC near half natural frequency electrostatically actuated MEMS cantilevers," Journal of Computational and Nonlinear Dynamics, vol. 8, pp. 031011-1-031011-16, 2013.

[20] D. I. Caruntu and M. Knecht, "On MEMS/NEMS biosensor sensitivity near half natural frequency," in Proceedings of the ASME 2010 International Design Engineering Technical Conferences and Computers and Information in Engineering Conference (IDETC/CIE '10), vol. 4, paper no. DETC2010-28612, pp. 439445, August 2010.

[21] D. I. Caruntu and M. Knecht, "On nonlinear response near-half natural frequency of electrostatically actuated microresonators," International Journal of Structural Stability and Dynamics, vol. 11, no. 4, pp. 641-672, 2011.

[22] W.-M. Zhang and G. Meng, "Nonlinear dynamic analysis of electrostatically actuated resonant MEMS sensors under parametric excitation," IEEE Sensors Journal, vol. 7, no. 3, pp. 370-380, 2007.

[23] W. Zhang and K. L. Turner, "Application of parametric resonance amplification in a single-crystal silicon micro-oscillator based mass sensor," Sensors and Actuators A: Physical, vol. 122, no. 1, pp. 23-30, 2005.

[24] J. F. Rhoads, S. W. Shaw, K. L. Turner, J. Moehlis, B. E. DeMartini, and W. Zhang, "Generalized parametric resonance in electrostatically actuated microelectromechanical oscillators," Journal of Sound and Vibration, vol. 296, no. 4-5, pp. 797-829, 2006.

[25] M.-H. Hsu, "Electromechanical analysis of electrostatic nanoactuators using the differential quadrature method," Communications in Numerical Methods in Engineering, vol. 24, no. 11, pp. 1445-1457, 2008.

[26] D. I. Caruntu and M. Knecht, "On nonlinear response of capacitive sensors," in Proceedings of the 2009 ASME International Design Engineering Technical Conferences and Computers and Information in Engineering Conference (DETC '09), vol. 6, pp. 531-538, September 2009.
[27] D. I. Caruntu and I. Martinez, "Reduced order model of parametric resonance of electrostatically actuated MEMS cantilever resonators," International Journal of Non-Linear Mechanics, 2014.

[28] D. I. Caruntu, "Dynamic modal characteristics of transverse vibrations of cantilevers of parabolic thickness," Mechanics Research Communications, vol. 36, no. 3, pp. 391-404, 2009.

[29] D. I. Caruntu, "Classical Jacobi polynomials, closed-form solutions for transverse vibrations," Journal of Sound and Vibration, vol. 306, no. 3-5, pp. 467-494, 2007.

[30] D. I. Caruntu, "Self-adjoint differential equations for classical orthogonal polynomials," Journal of Computational and Applied Mathematics, vol. 180, no. 1, pp. 107-118, 2005.

[31] D. I. Caruntu, "Factorization of self-adjoint ordinary differential equations," Journal of Applied Mathematics and Computation, vol. 219, pp. 7622-7631, 2013.

[32] D. I. Caruntu, "Eigenvalue singular problem of factorized fourth-order self-adjoint differential equations," Journal of Applied Mathematics and Computation, vol. 224, pp. 603-610, 2013.

[33] C. M. Bender and S. A. Orszag, Advanced Mathematical Methods for Scientists and Engineers, McGraw-Hill Book Company, 1978.

[34] B. P. Mann and N. D. Sims, "Energy harvesting from the nonlinear oscillations of magnetic levitation," Journal of Sound and Vibration, vol. 319, no. 1-2, pp. 515-530, 2009.

[35] P. F. Pai and A. H. Nayfeh, "Non-linear non-planar oscillations of a cantilever beam under lateral base excitations," International Journal of Non-Linear Mechanics, vol. 25, no. 5, pp. 455474, 1990.

[36] B. Pratiher, "Non-linear response of a magneto-elastic translating beam with prismatic joint for higher resonance conditions," International Journal of Non-Linear Mechanics, vol. 46, no. 5, pp. 685-692, 2011.

[37] Z. H. Feng, X. J. Lan, and X. D. Zhu, "Principal parametric resonances of a slender cantilever beam subject to axial narrowband random excitation of its base," International Journal of Non-Linear Mechanics, vol. 42, no. 10, pp. 1170-1185, 2007.

[38] L. D. Zavodney and A. H. Nayfeh, "The non-linear response of a slender beam carrying a lumped mass to a principal parametric excitation: theory and experiment," International Journal of Non-Linear Mechanics, vol. 24, no. 2, pp. 105-125, 1989.

[39] M. Sayed and M. Kamel, "Stability study and control of helicopter blade flapping vibrations," Applied Mathematical Modelling, vol. 35, no. 6, pp. 2820-2837, 2011.

[40] H. W. Rong, X. D. Wang, W. Xu, and T. Fang, "Saturation and resonance of nonlinear system under bounded noise excitation," Journal of Sound and Vibration, vol. 291, no. 1-2, pp. 48-59, 2006.

[41] L. N. Panda and R. C. Kar, "Nonlinear dynamics of a pipe conveying pulsating fluid with combination, principal parametric and internal resonances," Journal of Sound and Vibration, vol. 309, no. 3-5, pp. 375-406, 2008. 

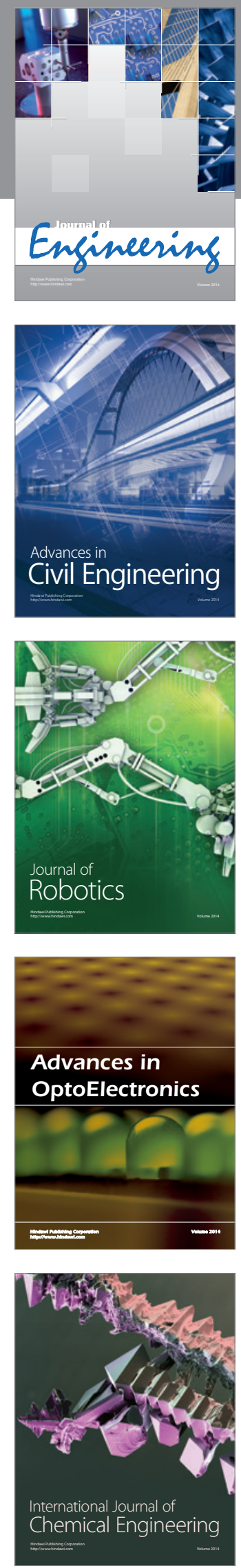

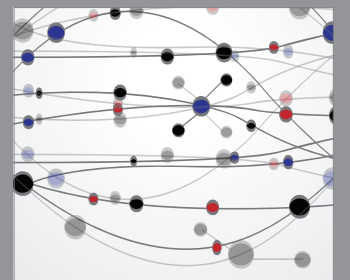

The Scientific World Journal
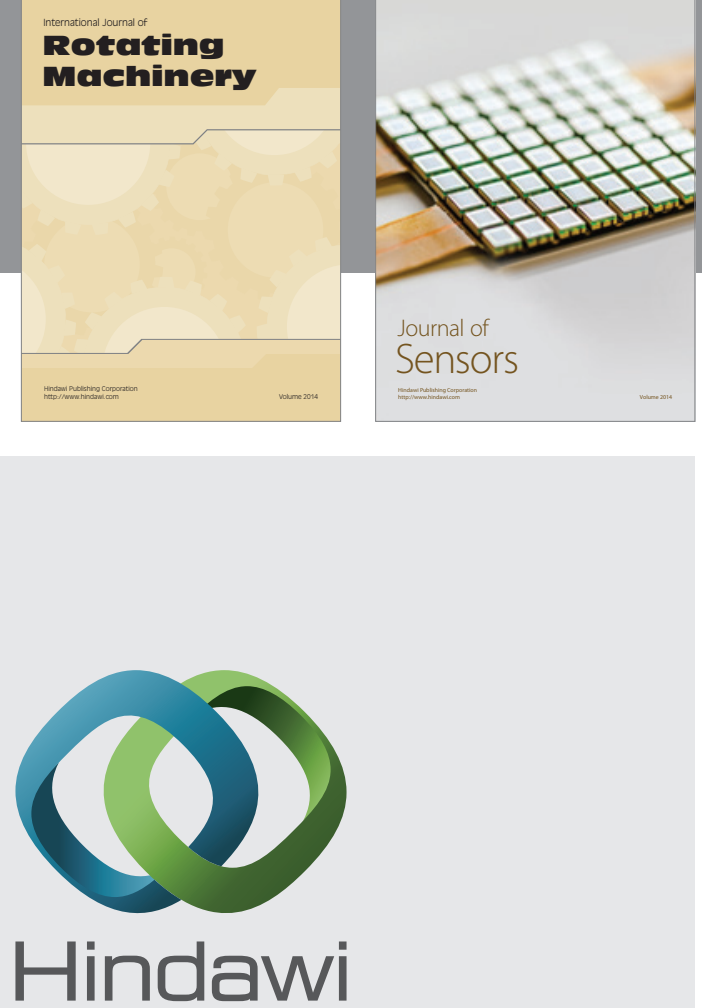

Submit your manuscripts at http://www.hindawi.com
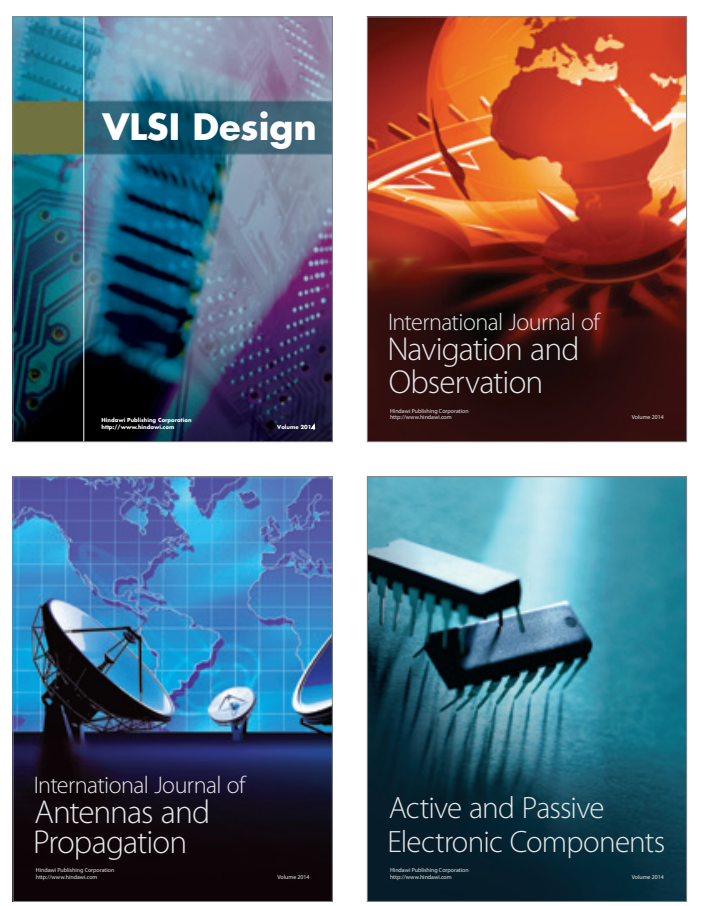
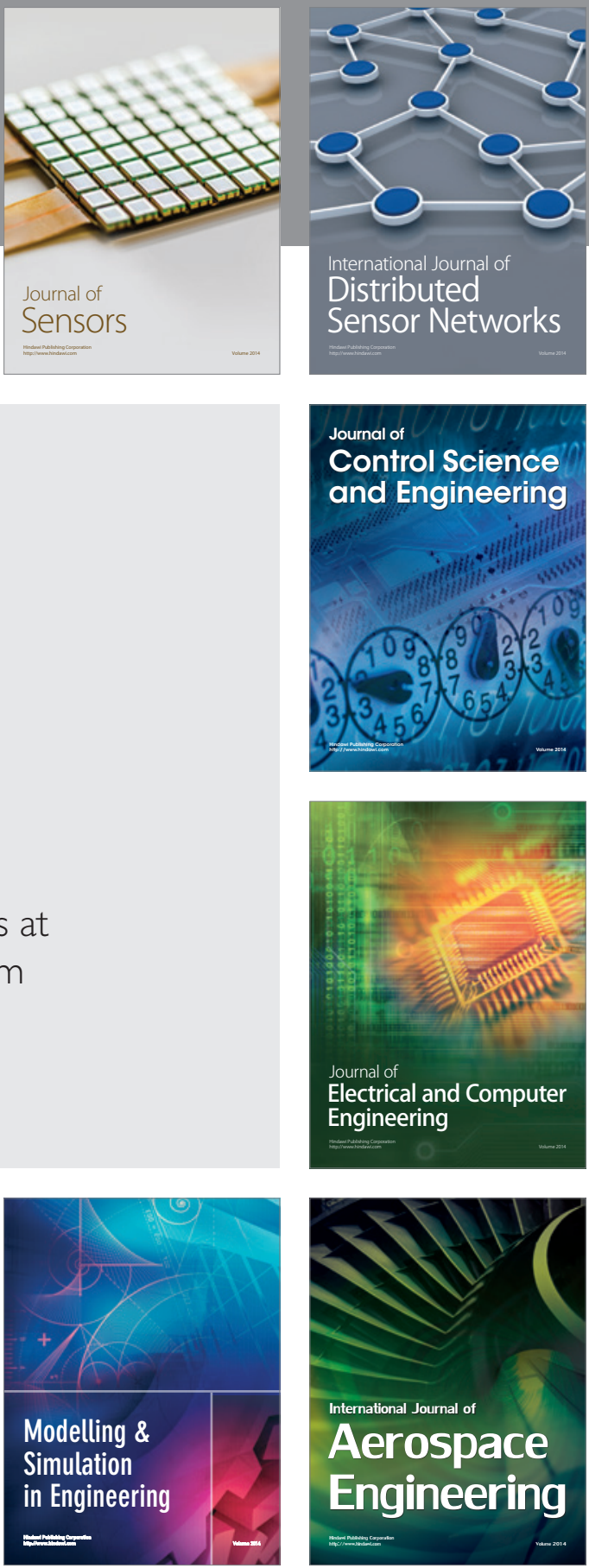

Journal of

Control Science

and Engineering
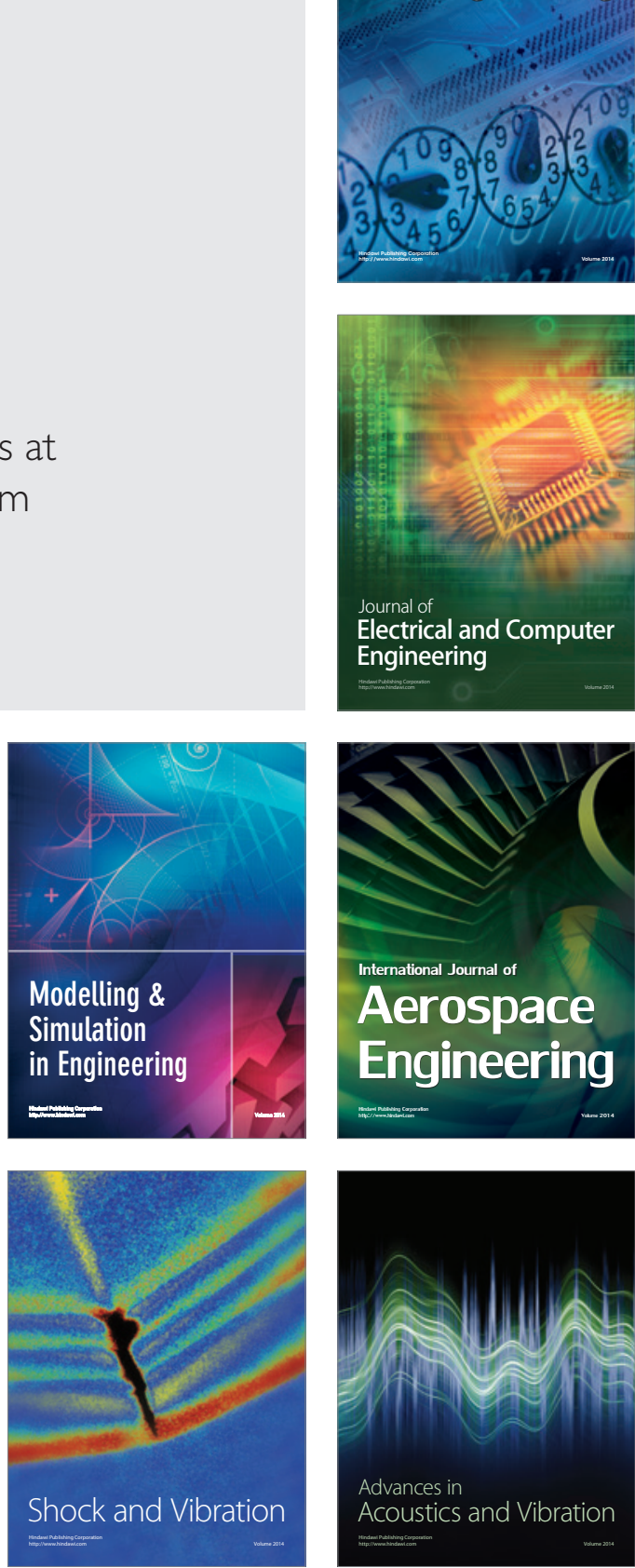